\title{
Profiles of Coronary Artery Disease Risk in Cardiac Patients: Actual versus Perceived
}

\author{
JULIE A BORSACK, STEVE MARTIN, KATHY L HILL, THOMAS MEADE, BECKY \\ BRADEN, IVAN PEREIRA, STEPHEN F CROUSE, \& JOHN S GREEN
}

Texas A\&M University, Sam Houston State University, Scott \& White Clinic



